\title{
Measurement of visual memory span by means of the recall of dot-in-matrix patterns
}

\author{
SHIN-ICHI ICHIKAWA \\ University of Tokyo, Bunkyo-ku, Tokyo 113, Japan
}

\begin{abstract}
A method for the study of visual memory span is described. The results of two dot-in-matrix experiments indicated that the patterns of larger matrix size were more difficult to recall, but the effect of visual angle was not significant. Visual memory span could be represented by the number of dots in the 5 by 5 or 6 by 6 pattern that a subject perfectly recalled with a probability of .5 , since the proportion of recalled patterns was a monotonic decreasing function of the number of dots. This method can be used in group experiments without consideration of the differences in visual angles caused by the locations of subjects. The selection of appropriate presentation times and the distinction between verbal and visual memory spans are discussed.
\end{abstract}

Klemmer and Frick (1953) and Attneave (1955) required subjects to recall positions of dots in a matrix in order to quantify the amount of transmitted information. This paper provides a simpler method to measure visual memory span. Visual memory span is represented by the number of dots in a pattern that is perfectly recalled with a probability of .5 , provided that the matrix size is appropriate and the arrangement of dots is suitably controlled.

Verbal memory span was originally defined by Woodworth (1938) as the length of the digit sequence that a subject can recall with a probability of .5 . This kind of memory span has been measured by the method of limits and the method of constant stimuli, with the assumption that the proportion correct for a digit sequence is a monotonic decreasing function of its length, that is, the number of digits in a sequence (Guilford \& Dallenbach, 1925; Oberly, 1928). Such measurement procedures take more time than the popular method in which an experimenter presents a stimulus longer than the span, which is then defined as the number of correctly recalled digits. This recall method, however, is often an inadequate way of measuring the maximum amount of information one can recall.

For visual memory span, we must find a satisfactory measure similar to the length of a digit sequence in measuring verbal memory span. With dot-in-matrix patterns, three factors affect recall: (1) matrix size (the numbers of rows and columns), (2) the number of dots in a matrix, and (3) the arrangement of dots. A pattern of larger matrix size is more difficult to recall than a pattern of smaller size, other factors being constant. However, matrix size alone may be an insufficient measure of

The author would like to thank Dr. T. Oyama of the University of Tokyo and Dr. C. Izawa of Tulane University for their many helpful comments. The author is now associated with Saitama University. Requests for reprints should be sent to Shin-ichi Ichikawa, Junior College of Economics, Saitama University, 255 Shimo-ohkubo, Urawa 338, Japan. visual memory span. The number of dots may be a preferable measure. In Experiment 1, both matrix size and number of dots are varied and the proportion of correctly recalled patterns is measured; the arrangement of dots is controlled by the method outlined below.

Random arrangement of dots in a matrix often results in varied levels of difficulty in recall. Therefore, some objective restrictions are needed to produce homogeneous stimuli. Ichikawa (1981) asked 40 subjects to rate 60 4 by 4 matrix patterns, each containing eight dots, for ease of memorization on a 9-point scale and performed multiple regression analysis. The criterion variable was the rated ease of memorization (REM) of the patterns, and the predictor of variables were 25 kinds of physical variables that described the configuration of dots in a matrix. As a result of the forward selection procedure, two variables were considered to be very effective for the prediction of REM.

1. Equivalence set size (ESS) is the number of patterns resulting from reflection and rotation in $90-\mathrm{deg}$ steps. The original pattern itself is counted as one. Generally, the more symmetric the pattern, the smaller the ESS value. This variable was originally proposed by Garner and Clement (1963).

2. Concentration along row or column direction (CRC) is a function of CR and CC. CR represents the degree of concentration of dots along row direction. Let $d_{i}$ be the number of dots contained in row $i(1 \leqslant i \leqslant m)$, and let $p_{i}=d_{i} / d$, where $d$ is the total number of dots in a matrix. Then,

$$
\mathrm{CR}=1-\left[\sum_{i=1}^{m} p_{i} \log _{2}\left(1 / p_{i}\right)\right] / \log _{2} m
$$

and if $p_{i}=0, p_{i} \log _{2}\left(1 / p_{i}\right)=0$. A larger CR means that dots are more concentrated in particular rows. Thus, CR derives from "redundancy" in information theory. In a similar manner, $\mathrm{CC}$ represents the degree of concentration of dots along column direction. CRC is 
defined as $\mathrm{CRC}=1-(1-\mathrm{CR})(1-\mathrm{CC})$. Accordingly, $\mathrm{CRC}$ increases with the increase in $\mathrm{CR}$ or $\mathrm{CC}$, and it becomes 1 if either CR or CC becomes 1 .

The very small correlation coefficient between ESS and CRC $(\mathrm{r}=-.181)$ indicates mutual independence of these measures. When these two variables are used for predicting $R E M$, the following multiple regression equation is obtained: REM est $=-.692$ ESS $^{*}+.385$ CRC* . Here, an asterisk indicates that the values of the variable are standardized so that the mean is zero and the variance is one, and $\mathrm{REM}_{\mathrm{e}}^{*}$ st represents the estimated value (leastsquare approximation) for REM*. The fairly large multiple correlation coefficient $(r=.850)$ seems to indicate success in prediction of REM by these two variables. In addition, immediate and free recall are correlated with the values estimated by the equation. Thus, it appears that arrangement of dots can be controlled by ESS and $\mathrm{CRC}$ in recall experiments.

\section{EXPERIMENT 1}

In this experiment, the matrix size and number of dots were varied. It was predicted that the proportion of correctly recalled patterns was a monotonic decreasing function of the number of dots, provided that the matrix size was appropriate and the arrangement of dots was well controlled. If this prediction is verified, we will employ the number of dots as a measure to express visual memory span.

\section{Method}

Subjects. The subjects were 10 undergraduate students ( 7 males and 3 females), ages 21 to 25 years.

Materials. Five sets of dot-in-matrix patterns were used. Each set consisted of 38 patterns that were different in matrix size and in the number of dots, as shown in Table 1. The number of dots never exceeded half the number of cells in a matrix. Otherwise, subjects could recall the patterns by memorizing blank cells instead of filled ones.

The arrangement of dots was controlled by the following two general restrictions: ESS should be eight and CRC should be smaller than the value of the mean of 50 randomly produced patterns minus their standard deviation. The mean and the standard deviation for each type of pattern are shown in Table 2. Note that the above two restrictions cannot always be satisfied when the population of possible patterns is too small ( 3 by 3 patterns or patterns which contain one or two dots in a 4 by 4 , 5 by 5 , or 6 by 6 matrix). In such cases, patterns were selected so that ESS was maximized and CRC was minimized.

Each pattern was drawn on a white card. The cell size was $1 \times 1 \mathrm{~cm}$, and the diameter of a dot was $5 \mathrm{~mm}$. Some examples are shown in Figure 1.

Table 1

Matrix Size and Number of Dots of the Patterns Used in Experiment 1

\begin{tabular}{cc}
\hline Matrix Size & \multicolumn{1}{c}{ Number of Dots } \\
\hline 3 by 3 & $1,2,3,4$ \\
4 by 4 & $1,2,3,4,5,6,7,8$ \\
5 by 5 & $1,2,3,4,5,6,7,8,9,10,11,12$ \\
6 by 6 & $1,2,3,4,5,6,7,8,9,10,12,14,16,18$ \\
\hline
\end{tabular}

Table 2

Means and Standard Deviations of CRC Values for 50 Randomly Produced Patterns

\begin{tabular}{|c|c|c|}
\hline Number of Dots & Mean & $\mathrm{SD}$ \\
\hline \multicolumn{3}{|c|}{3 by 3 Matrix } \\
\hline 1 & 1.0000 & .0000 \\
\hline 2 & .7771 & .1976 \\
\hline 3 & .5925 & .1889 \\
\hline 4 & .3290 & .1987 \\
\hline \multicolumn{3}{|c|}{4 by 4 Matrix } \\
\hline 1 & 1.0000 & .0000 \\
\hline 2 & .8650 & .1246 \\
\hline 3 & .6368 & .1517 \\
\hline 4 & .4863 & .1575 \\
\hline 5 & .3391 & .1330 \\
\hline 6 & .2649 & .1376 \\
\hline 7 & .2225 & .1104 \\
\hline 8 & .1617 & .0845 \\
\hline \multicolumn{3}{|c|}{5 by 5 Matrix } \\
\hline 1 & 1.0000 & .0000 \\
\hline 2 & .8702 & .0850 \\
\hline 3 & .7179 & .1178 \\
\hline 4 & .5743 & .1056 \\
\hline 5 & .4513 & .1093 \\
\hline 6 & .4115 & .1300 \\
\hline 7 & .3325 & .0947 \\
\hline 8 & .2525 & .1021 \\
\hline 9 & .2061 & .0816 \\
\hline 10 & .1601 & .0699 \\
\hline 11 & .1562 & .0759 \\
\hline 12 & .1285 & .0579 \\
\hline \multicolumn{3}{|c|}{6 by 6 Matrix } \\
\hline 1 & 1.0000 & .0000 \\
\hline 2 & .8893 & .0656 \\
\hline 3 & .7268 & .1017 \\
\hline 4 & .6180 & .1291 \\
\hline 5 & .5239 & .1199 \\
\hline 6 & .4326 & .1375 \\
\hline 7 & .3685 & .0943 \\
\hline 8 & .3269 & .1106 \\
\hline 9 & .2690 & .0996 \\
\hline 10 & .2393 & .0817 \\
\hline 12 & .1874 & .0699 \\
\hline 14 & .1483 & .0691 \\
\hline 16 & .1065 & .0503 \\
\hline 18 & .0937 & .0391 \\
\hline
\end{tabular}

Apparatus. A Ralph Gerbrands tachistoscope (Model T-4A) was used for presenting materials. The distance from the subject's eye to the stimulus field was $83 \mathrm{~cm}$; the size of a matrix cell became $.69 \times .69 \mathrm{deg}$. The luminance of the field was $40 \mathrm{~cd} / \mathrm{m}^{2}$.

Procedure. A blank card was presented for $1 \mathrm{sec}$ as a signal. One second after the signal disappeared, a stimulus pattern was presented for $1 \mathrm{sec}$. The subject was required to reproduce the pattern immediately after the presentation by marking the positions of dots in a grid (the cell size was $5 \times 5 \mathrm{~mm}$ ) on an answer sheet with a pen. When the subject finished recall, the next trial began.

Each subject was tested on all five sets of stimulus patterns, and the order of sets was randomized from subject to subject. The presentation order in one set was as follows: $1,2,3,4$ dots in 3 by $3 ; 1,2, \ldots, 8$ dots in 4 by $4 ; 1,2, \ldots, 12$ dots in 5 by $5 ; 1,2, \ldots, 18$ dots in 6 by 6 . Every subject was informed of both the number of dots in a pattern before the presentation of it and the matrix size prior to a change. The sub- 

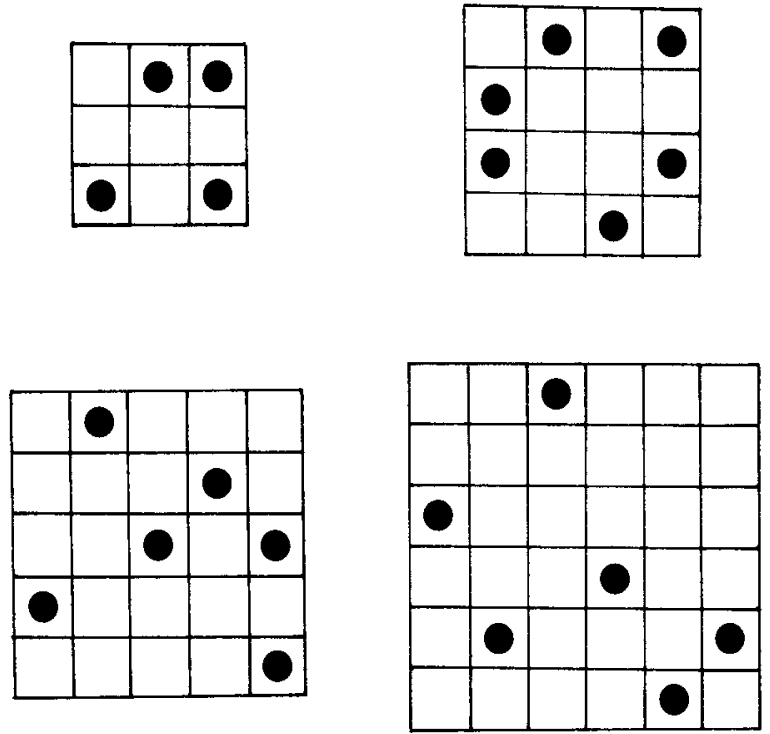

Figure 1. Examples of dot-in-matrix patterns used in Experiment 1 .

jects were urged to recall as many dots as possible. An experimental session required approximately $50 \mathrm{~min}$.

\section{Results and Discussion}

Figure 2 shows the pooled proportion of the patterns recalled perfectly as a function of the number of dots. The following two characteristics are found in the curve for 5 by 5 and 6 by 6 patterns: The proportion correct is a monotonic decreasing function of the number of dots, and the curve passes with a considerably steep slope through the $50 \%$ correct level. These results suggest that visual memory span can be represented in terms of the number of dots for each matrix size. That is, we can define the visual memory span of a subject as the number of dots in the pattern which can be fully recalled with a probability of .5 .

This measure made it possible to compare the abilities of subjects or the effects of experimental conditions. In fact, the visual memory spans of the 10 subjects were calculated from their individual performances. The summation method (Guilford, 1954) was adopted for computation. Two values of spans for each subject were obtained from the data for 5 by 5 and 6 by 6 patterns, respectively. Figure 3 is a scattergram of these values. The abscissa represents the span for 5 by 5 patterns and the ordinate, for 6 by 6 patterns. The considerably high correlation suggested that these values had high reliability, although each subject was tested on only five series.

However, recall was always perfect for 3 by 3 patterns irrespective of the number of dots. For 4 by 4 patterns, the proportion correct slightly decreased with the increase in the number of dots, but was always higher than the $50 \%$ level. Consequently, these patterns cannot be employed to measure visual memory span, at least for presentations longer than $1 \mathrm{sec}$.

Finally, recall difficulty apparently increased with matrix size as was expected, even when the number of dots was kept constant. It is, however, possible to ascribe this effect to the visual angles of the patterns instead of the matrix size in a mathematical sense. A pattern of large matrix size necessitated a large visual angle, since the length of the side of each cell was fixed at $1 \mathrm{~cm}(.69 \mathrm{deg})$. Accordingly, subjects had to make faster visual scans to see the entire matrix than when they observed a pattern of small matrix size. This may account for the results of Experiment 1. In Experiment 2, the effect of the matrix size and that of visual angle upon recall performance are investigated through a factorial design.

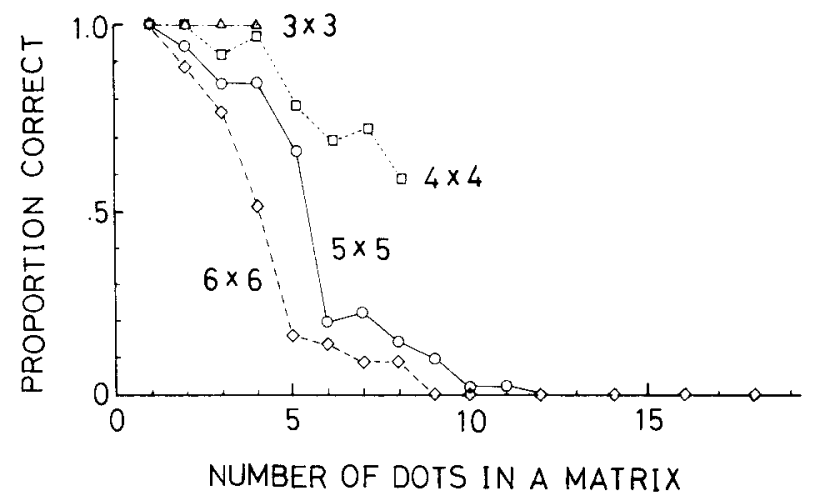

Figure 2. Proportion of the number of patterns correctly recalled after $1 \mathrm{sec}$ presentations as a function of matrix size and number of dots (Experiment 1).

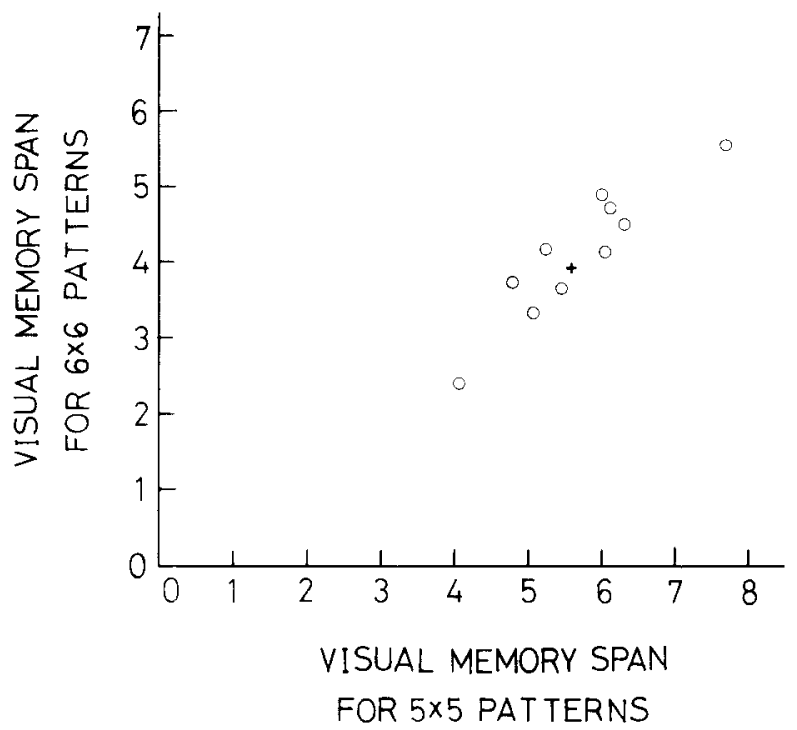

Figure 3. A scattergram of 10 subjects' visual memory spans measured by the recall of 5 by 5 and 6 by 6 patterns, respectively. The plus indicates the mean value (Experiment 1). 


\section{EXPERIMENT 2}

\section{Method}

Subjects. A new set of 10 undergraduate students participated: 8 males and 2 females, ages 20 to 25 years.

Materials. Three levels of matrix size and three levels of visual angle produced nine patterns in one set. The matrix sizes were 4 by 4,5 by 5 , and 6 by 6 . The visual angles of the whole matrix were $2.07 \times 2.07 \mathrm{deg}$ (called small condition), 3.11 by $3.11 \mathrm{deg}$ (called medium), and 4.14 by 4.14 deg (called large). The range of the visual angles corresponded to that in Experiment 1 . The number of dots contained in a matrix was five in all nine conditions. The diameter of a dot was always half of the horizontal length of each matrix cell. The arrangement of dots was again controlled by ESS and CRC. One set for practice and five sets for experimental sessions were prepared.

Procedure. Each pattern was drawn on a white card and presented through the same tachistoscope with procedures similar to those used in Experiment 1. The subjects reproduced the stimulus patterns on answer sheets where grids (the cell size was $5 \times 5 \mathrm{~mm}$ ) were drawn. Following a practice session, each subject participated in five experimental sessions, with each session containing nine conditions. Presentation order was randomized from subject to subject by shuffling the stimulus cards. Experimental sessions for each subject required about $20 \mathrm{~min}$.

\section{Results and Discussion}

As shown in Table 3, the proportion of correctly reproduced patterns was not largely influenced by visual angle, but was greatly affected by matrix size. An analysis of variance for repeated measures (matrix size by visual angle by subjects) was carried out for the number of correctly recalled patterns. The main effect of matrix size was significant $[F(2,18)=139.49, p<.001]$. Neither the visual angle nor the matrix size by visual angle interaction was statistically significant. Therefore, we cannot attribute the effect of matrix size shown in Experiment 1 to the visual angle which changed proportionally with matrix size.

Since visual angle appears unimportant, the method can be used in group experiments without distorting data. If the visual angle were effective, we would have to consider the distance between a stimulus field and a subject's seat in comparing scores of the subjects.

\section{GENERAL DISCUSSION}

Experiment 1 showed that the proportion correct for 5 by 5 or 6 by 6 patterns is a monotonic decreasing function of the number of dots contained therein, and

Table 3

Proportion of Correctly Recalled Patterns as a Function of Matrix Size and Whole Visual Angle (Experiment 2)

\begin{tabular}{ccccc}
\hline $\begin{array}{c}\text { Matrix } \\
\text { Size }\end{array}$ & Small & Medium & Large & Mean \\
\hline 4 by 4 & .86 & .88 & .88 & .873 \\
5 by 5 & .54 & .48 & .48 & .500 \\
6 by 6 & .12 & .18 & .20 & .167 \\
Mean & .507 & .513 & .520 & .513 \\
\hline
\end{tabular}

that the curve passes with a steep slope though the $50 \%$ level. Because of these two characteristics, we can define visual memory span in terms of the number of dots in the pattern that a subject completely recalls with a probablility of .5 .

To this point, two important problems have not been discussed. First, how long should we present a dot pattern? Generally speaking, immediate memory span refers to a limitation on memory rather than on perception or visual scanning. In measuring verbal memory span, digits are presented one after another slowly enough for a subject to read each of them. Then typical subjects recall seven plus or minus two digits (Miller, 1956). When simultaneous instead of successive presentation is adopted, we must employ an appropriate exposure duration. If the duration is too short, the rate of visual scanning rather than the capacity of memory per se may be an important factor. However, rehearsal and encoding processes apparently increase memory span if the stimulus is presented too long. Ichikawa (Note 1) plotted verbal and visual spans as functions of their presentation times ranging from $1 \mathrm{msec}$ to $64 \mathrm{sec}$, using digit sequences and 5 by 5 patterns, respectively. The span at each presentation time was measured through the method of limits. Both curves had similar features: Each increased rapidly as long as presentation time was shorter than $1 \mathrm{sec}$, but there was a plateau at the 2 to 8 -sec presentation levels. The height of this plateau, which seems to indicate so-called "immediate memory span," is about nine digits and six dots. The spans increased again if the presentation time became longer than $16 \mathrm{sec}$. Thus, presentation times between 1 and $10 \mathrm{sec}$ seem adequate in measuring memory span.

The second problem concerns whether or not the reproduction of dot-in-matrix patterns is an appropriate procedure to measure "visual" memory span. One may assume that subjects could verbally encode and retain the positions of dots in a matrix. If this is true, the recall of dot patterns cannot be referred to as "visual" memory. There are two arguments against such an idea: (1) The retention of letters or digits was interfered with substantially by verbal, but not so much by visual, distracting tasks. For the retention of positions of items in a matrix, on the other hand, the opposite holds true (den Heyer \& Barrett, 1971; Meudell, 1972; Ichikawa, Note 1). (2) The correlation coefficient between verbal and visual memory spans, measured by the recall of digit sequences and by dot patterns, respectively, is very small $(\mathrm{r}=.23$; Ichikawa, Note 1$)$. This strongly suggests that the position of information is retained in a form other than that of verbal information.

\section{CONCLUSIONS}

In the present paper, a basis was presented to measure visual memory span through the method of constant stimuli or the method of limits, using dot-in-matrix patterns as test materials. These methods are apparently 
laborious but often give highly reliable values. This kind of measurement may be useful in providing insights into the mechanisms of human memory, the contribution of visual imagery process to thinking, and other related problems.

\section{REFERENCE NOTE}

1. Ichikawa, S. Independence of verbal and visual short-term storages shown in the recall of digit sequences and dot-in-matrix patterns. Unpublished manuscript, 1981.

\section{REFERENCES}

Attneave, F. Symmetry, information, and memory for patterns. American Journal of Psychology, 1955, 68, 209-222.

DEN HEYER, K., \& BARRETT, B. Selective loss of visual and verbal information in STM by means of visual and verbal interpolated tasks. Psychonomic Science, 1971, 25, 100-102.

Garner, W. R., \& Clement, D. E. Goodness of pattern and pattern uncertainty. Journal of Verbal Learning and Verbal Behavior, 1963, 2, 446-452.

Guilford, J. P. Psychometric methods. New York: McGrawHill, 1954.
Guilford, J. P., \& Dallenbach, K. M. The determination of memory span by the method of constant stimuli. American Journal of Psychology, 1925, 36, 621-628.

ICHIXAWA, S. Rated ease of memorization of dot-in-matrix patterns: Multiple regression analysis by physical variables which describe configuration of dots. Japanese Psychological Research, 1981, 23, 69-78.

Klemmer, E. T., \& Frick, F. C. Assimilation of information from dot and matrix patterns. Journal of Experimental Psychology, 1953, 45, 15-19.

MeudeLL, P. R. Short-term visual memory: Comparative effects of two types of distraction on the recall of visually presented verbal and nonverbal material. Journal of Experimental Psychology, 1972, 94, 244-247.

Miller, G. A. The magical number seven, plus or minus two: Some limits on our capacity for processing information. Psychological Review, 1956, 63, 81-97.

Oberly, H. S. A comparison of the spans of "attention" and memory. American Journal of Psychology, 1928, 40, 295-302.

Woodworth, R. S. Experimental psychology. New York: Holt, Rinehart, \& Winston, 1938.

(Received for publication July 10, 1981; revision accepted January 14,1982 .) 